\title{
30
}

\section{Self-independent Petri Nets for Distributed Systems*}

\author{
Yong SUN ${ }^{\dagger}$, Shaoying $\mathrm{LIU}^{\star}$, Mitsuru OHBA* \\ ${ }^{\dagger}$ Department of Computer Science \\ The Queen's University of Belfast \\ Belfast BT7 1NN, Northern Ireland \\ Email: Y.Sun@qub.ac.uk \\ *Faculty of Information Sciences \\ Hiroshima City University \\ Asaminami-ku, Hiroshima, 731-31, Japan \\ Email: \{shaoying, ohba \}@cs.hiroshima-cu.ac.jp
}

\begin{abstract}
We propose that asynchronous mechanism for communication should be considered as a primitive for semantic models of distributed systems in contrast to the synchronous mechanism (hand-shaking) of CSP (Hoare, 1985) and of CCS (Milner, 1989). By doing so, we present an approach to producing a deadlock-free system, and give a denotational model for CCS in a non-interleaving fashion.
\end{abstract}

\section{Keywords}

asynchronous communication, self-independence, Petri nets, CCS, pre-order, partial order, complete partial order (cpo), denotational semantics

\section{Introduction and Overview}

Hand-shaking is an abstract and ideal synchronous mechanism for communication. It is adopted by Hoare in CSP (1985) and by Milner in CCS (1989) as a primitive to describe communications between different components in distributed systems. However, there is a synchronization problem associated with the implementation of this mechanism (Lamport et al, 1982; Sun \& Yang, 1996). Asynchronous communication is inevitable in the implementation of distributed and concurrent systems. The conventional way to cope with this problem is to simulate asynchronous communication using the hand-shaking mechanism. However, such a simulation is often awkward and inconvenient. Furthermore, the hand-shaking mechanism may result in deadlock; in our proposed asynchronous communication this will not occur. For instance,

$$
\alpha ? x . \beta ? y . B(x, y) \| \beta ! e_{1} . \alpha ! e_{2} . C
$$

*Work is supported in part by the Ministry of Education of Japan under Joint Research Grant-inAid for International Scientific Research (08044167), by Hiroshima City University under Hiroshima City University Grant for Special Academic Research (International Studies) SCS-FM (A440), and by NIDevR of the Queen's University of Belfast. 
in CSP and

$$
\left(\alpha x . \beta y . B(x, y) \mid \bar{\beta} e_{1} . \bar{\alpha} e_{2} . C\right) \backslash \alpha \beta
$$

in CCS are two examples of deadlocked processes (or deadlocked agents). They both describe that the left agent of the operator $\|$ (or |) synchronizes with the right agent. The left agent performs an $\alpha$ action (receiving) followed by a $\beta$ action (receiving) and then behaves like $B(x, y)$. The right agent performs a $\beta$ action (sending) followed by an $\alpha$ action (sending) and then behaves like $C$. Since the order of the actions in one agent does not match that in the other agent, deadlock is caused in synchronous communication. Such occurrences should not arise in the design of distributed systems. If a complete inference rule system existed which could be used to decide whether a deadlock exists in a communicating system, then we would use this system to exclude all deadlocks. Unfortunately, such a system cannot be constructed since the decision problem for deadlock is undecidable.

In order to solve the problems mentioned above, we propose that an asynchronous mechanism for communication be used as a primitive for semantic models of distributed systems, say CCS. Thus, as we will show, hand-shaking communication becomes a special case of asynchronous communication. Because of the asynchronous communication, every process (or agent) is self-independent which prevents deadlocks in distributed systems. That is, deadlock-freedom is a property inherited from the asychronous communication rather than resulting from an intricate matching of the orders of communicating actions among distributed processes (or agents).

In this paper, we first replace the synchronous mechanism by the asynchronous mechanism using specialized Petri nets (Petri, 1976; Peterson, 1981). This new kind of Petri net is called a self-independent Petri net. Such Petri nets provide a denotational model for CCS in a non-interleaving fashion (or so-called true concurrency). However, we omit the interpretation of CCS terms in the model since it is the same as that in the CCS semantics model. The reader may consult Goltz and Mycroft (1984) or Winskel (1983) for details.

The model to be presented is described through labelled Petri nets in which all constructable nets are self-independent (or deadlock-free). The class of all constructable nets provides an appropriate and sufficiently abstract domain in which the fixpoints of semantic equations can be evaluated when the isomorphic nets are regarded as being identical. Technically, the result can be regarded as an improvement of the following:

1. Winskel's (1983) event structures in which non-determinism is non-symmetric (i.e. the semantics of $B_{1}+B_{2}$ is not the same as the semantics of $B_{2}+B_{1}$ );

2. Goltz and Mycroft's (1984) labelled Petri nets which can not systematically deal with recursive processes (i.e. they have a difficulty in giving a semantics to $B_{2}$ in $\left.\left\{B_{1} \Leftarrow \alpha . N i l\left|\gamma . B_{1}, B_{2} \Leftarrow B_{1}\right| \beta . B_{2}\right\}\right) ;$

3. Boudol and Castellani (1987) follow Winskel's approach to solve the problem of non-symmetric of non-determinism, but their results are limited to finite structures.

In summary, we demonstrate that asynchronoous systems have better deadlock free properties. We will give a class of Petri nets which preserve deadlock freeness. Such nets can be used as a CCS semantics model (Milner, 1989).

The remainder of this paper is organized as follows. Section 2 introduces Self-independent Petri Nets (SPNs). Section 3 describes constructable SPNs. In section 4, we discuss 
isomorphic classes of SPNs. Finally, we compare the newly introduced notation with existing notations, give some conclusions, and sketch some future work.

\section{Introduction to Self-independent Petri Nets (SPNs)}

A labelled Petri net (LPN or simply PN) $N$ can be denoted as

$$
<S, E, T, L>
$$

where $S$ is a set of states (or places), $E$ is a set of events, $T$ is a set of transitions which is a subset of $(S \times E) \cup(E \times S), L$ is a labelling from events $E$ to labels $L a b$. For the sake of simplicity, we let $N$ stand for $S \cup E$.

For $x \in N$, the pre- and post-set of $x$ are defined, respectively, as

$$
\begin{aligned}
& \text { 1. } \cdot x={ }_{\text {def }}\{y \in N \mid<y, x>\in T\} \text { and as } \\
& \text { 2. } x^{\bullet}=_{\text {def }}\{y \in N \mid<x, y>\in T\} .
\end{aligned}
$$

A root-set of net $N,{ }^{\circ} N$, is defined as ${ }^{\circ} N={ }_{\text {def }}\left\{\left.x \in N\right|^{\bullet} x=\emptyset\right\} .|X|$ stands for the cardinality of the set $X$. The roots of $N$ (i.e. the elements of ${ }^{\circ} N$ ) can be considered as being always initially marked, although markings are not discussed at all in this paper. This treatment can be viewed as presupposing that all roots (states) are always marked initially and that multi-tokens are linearized into multi-nets, i.e. every initial state represents one token, see the example in Figure 1.

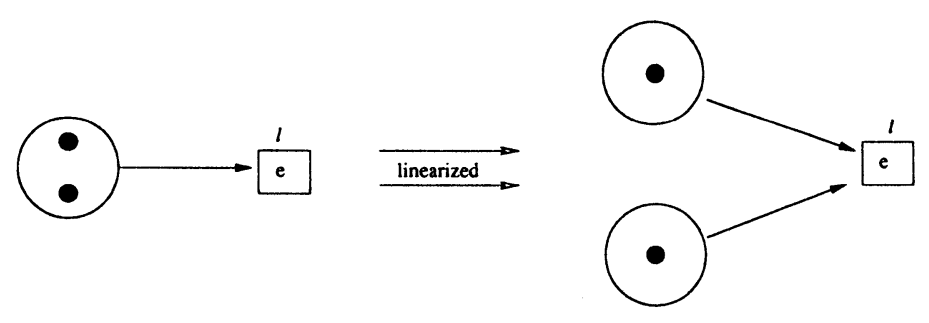

Figure 1: linearizing of tokens

For Petri nets, there are three phenomena which need to be classified.

1. The events $e_{1}$ and $e_{2}$ in Figure 2 are to be exclusively triggered, i.e. either but not both can be triggered.

In other words, these two events are not independent of each other. This phenomenon is commonly referred to as non-determinism.

2. The states $s_{1}$ and $s_{2}$ in Figure 3 are independent of each other, i.e. both states $s_{1}$ and $s_{2}$ are triggered by the event $e$ at the same time.

This phenomenon is commonly referred to as concurrency or parallelism. 


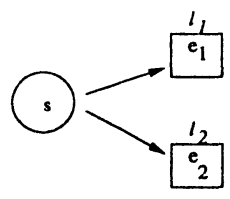

Figure 2: non-determinism

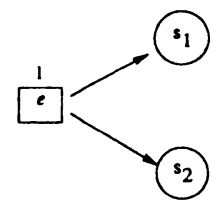

Figure 3: parallelism

3. The event $e$ in Figure 4 can only be triggered by both the states $s_{1}$ and $s_{2}$ (but not by only one of them).

This phenomenon is commonly referred to as synchronization.

To exploit these phenomena further, we introduce a parallel operator / over nets. Consider the net $N_{1}$ in Figure 5 and the net $N_{2}$ in Figure 6.

Then, their composition $N_{1} \mid N_{2}$ is as shown in Figure 7.

In these diagrams (see Figures 5, 6 and 7), each horizontal arrow can roughly be viewed as an execution (or evolution) of a process, and non-horizontal arrows are interactions between processes. The events labelled by $\tau \mathrm{s}$ (say $e_{4}$ and $e_{5}$ ) are successful communicating actions, and the events labelled differently (say $e_{1}$ and $e_{2}$ ) indicate unsuccessful communicating actions. More specifically, $N_{1}$ in $N_{1} \mid N_{2}$ at $s_{2}$ does not know whether the action performed on port $\ell$ is a result of the event $e_{1}$ or a result of the event $e_{4}$ (or $e_{5}$ ), unless it checks the receiving message. That is, the result of an action has to be examined before $N_{1}$ understands whether it is a successful action. In other words, an empty message implies that the action was unsuccessful. Among successful actions, $N_{1}$ needs to be informed by $N_{2}$ which event produced the received message, say event $e_{4}$ or event $e_{5}$. Therefore, every communicating action takes place only subject to the performer's

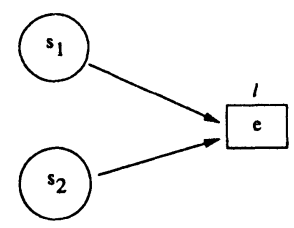

Figure 4: synchronization 


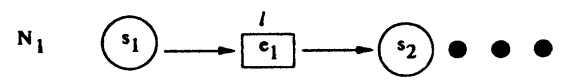

Figure 5: net $N_{1}$

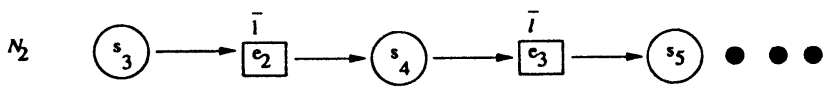

Figure 6: net $N_{2}$

wish regardless of:

1. who a message sender or a message receiver is, and

2. who the communicating partner is.

Synchronization is the result of a "pure coincidence" (i.e. the actions labelled by $l$ and its partner $\bar{l}$ can be executed independently). Therefore, synchrony is a special case of asynchrony.

We obtain two extra advantages from such asynchrony. These are:

1. every process is self-independent;

2. the message loss in communication is a natural consequence of the asynchronous communication.

Because of the latter, systems are able to tolerate message losses, which is an important feature in distributed computing systems. Actually, we say that a relation $\|$ on nets is the independent relation on nets, i.e. $\|:(S \times S) \cup(E \times E) \cup(S \times E) \cup(E \times S)$ is the least relation on net $N$ which satisfies the following six conditions:

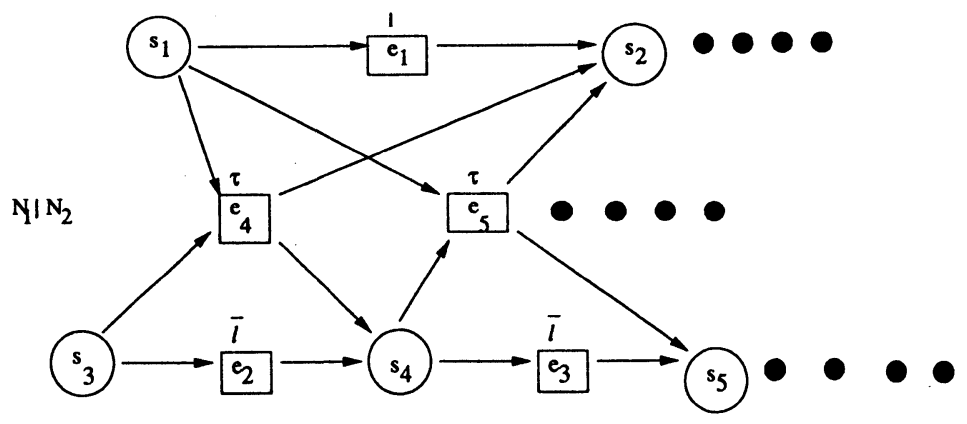

Figure 7: composition $N_{1} \mid N_{2}$ 
1. $\forall s_{1}, s_{2} \in{ }^{\circ} N . s_{1} \neq s_{2} \Rightarrow s_{1} \| s_{2}$;

2. $\forall e \in E . \forall s_{1}, s_{2} \in e^{\bullet} . s_{1} \neq s_{2} \Rightarrow s_{1} \| s_{2}$;

3. $\forall e_{1}, e_{2} \in E .\left(\left({ }^{\bullet} e_{1} \cap{ }^{\bullet} e_{2}=\emptyset\right) \wedge\left(\forall s_{1} \in^{\bullet} e_{1} \cdot \forall s_{2} \epsilon^{\bullet} e_{2} . s_{1} \| s_{2}\right)\right) \Rightarrow e_{1} \| e_{2} ;$

4. $\forall e_{1}, e_{2} \in E . e_{1} \| e_{2} \Rightarrow\left(\forall s_{1} \in{ }^{\bullet} e_{1} . s_{1} \| e_{2}\right) \wedge\left(\forall s_{2} \in e^{\bullet} e_{2} . e_{1} \| s_{2}\right)$;

5. $\forall s \in S . \forall e \in E .\left(\left(\forall s^{\prime} \in{ }^{\bullet} e . s \| s^{\prime}\right) \vee\left(\forall s^{\prime} \in{ }^{\bullet} e . s^{\prime} \| s\right)\right) \Rightarrow(s\|e \wedge e\| s)$;

6. $\forall s \in S . \forall e \in E .\left(s\left\|e \Rightarrow \forall s^{\prime} \in e^{\bullet} . s\right\| s^{\prime}\right) \wedge\left(e\left\|s \Rightarrow \forall s^{\prime} \in e^{\bullet} . s^{\prime}\right\| s\right)$.

The first condition states that the distinct initial states are independent of each other; the second condition states that the distinct states triggered by a same event are independent of each other; the third condition expresses the fact that the distinct events triggered by totally independent states are independent of each other; the fourth condition indicates that an event and a state are independent of each other if the state is triggered by another event independent of this event; the fifth condition states that an event and a state are independent of each other if all the states triggering the event are independent of the state; the sixth condition indicates that a state (say $s$ ) is independent of another state (say $s^{\prime}$ ) if the other state $\left(s^{\prime}\right)$ is triggered by an event which is independent of the state $(s)$. With the understanding of the independence relation on nets, we are interested in those nets which have the following six properties:

1. $\forall x, y \in N .\left(\bullet^{\bullet} x=\bullet y\right) \wedge\left(x^{\bullet}=y^{\bullet}\right) \Rightarrow x=y$;

2. $\forall e \in E . \exists s_{1}, s_{2} \in S . s_{1} T e \wedge e T s_{2}$;

3. II is irreflexive;

4. $\forall e \in E . \forall s_{1}, s_{2} \in{ }^{\bullet} e . s_{1} \neq s_{2} \Rightarrow s_{1} \| s_{2}$;

5. $\forall s \in S . \forall e_{1}, e_{2} \in \bullet s . \neg\left(e_{1} \| e_{2}\right)$;

6. $\forall s \in S-{ }^{\circ} N \cdot \bigcap\left\{{ }^{\bullet} e \mid e \in{ }^{\bullet} s\right\} \neq \emptyset$.

The first property states that the nets we are interested in have no redundancy; the second property expresses the fact that every event has a cause and a result in the nets; the third property states that either an event or a state cannot be independent of itself; the fourth property states that the distinct states triggering the same event must be independent of each other; the fifth property indicates that the distinct events triggering the same state cannot be independent of each other; the last property asserts that if a non-initial state is triggered by a collection of events, these events must share a common triggering state.

If, for any net $N$, the independence relation $\|$ on the nets has the above six properties, we say that $N$ is a self-independent (labelled) Petri net, or a SPN. Actually, the fourth property of SPNs excludes the nets such as that in Figure 8 and the fifth property excludes the nets such as that in Figure 9. These exclusions are reasonable, i.e. nondeterminism should not lead to synchronization; and concurrency (or parallelism) should not be reduced to non-determinism.

It is worth mentioning that $\|$ is a reversed version of \# (the conflict relation) in Neilsen et al (1980), but SPNs are richer than the nets described there since they (i.e. SPNs) are 


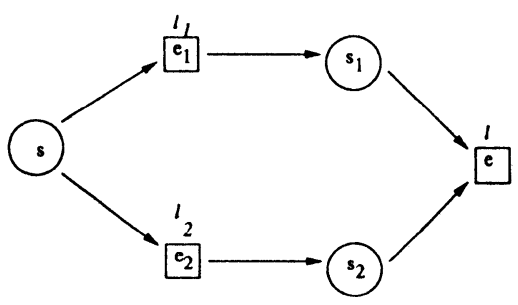

Figure 8: non-determinism $\rightarrow$ synchronization

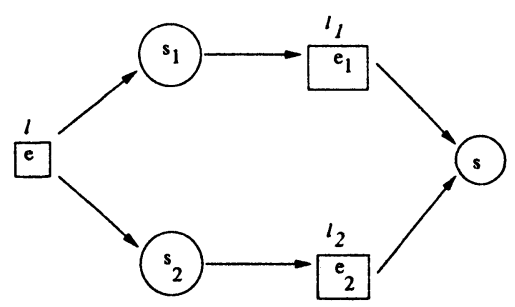

Figure 9: parallelism $\rightarrow$ non-determinism

not necessarily cycle-free. However, the existence of a cycle in a net is closely related to a deadlock in the net. An exmaple with a cycle is shown in Figure 10. Fortunately, this does not happen in the constructable nets with which this paper is concerned.

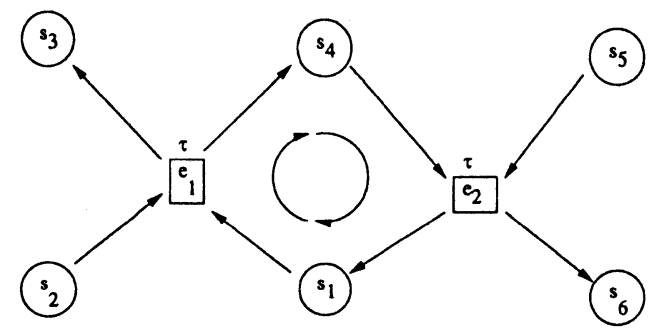

Figure 10: a circle in a net (a deadlock)

We are not interested in the trivial SPN, i.e. $\langle\emptyset, \emptyset, \emptyset, \emptyset\rangle$ is not included inside our SPNs. Also, since we intend to regard isomorphic nets as identical, we treat all the singleton nets as the same net and write it as $\langle\{\bullet\}, \emptyset, \emptyset, \emptyset\rangle$. For simplicity, we assume that the roots of each net are states, i.e. ${ }^{\circ} N \subseteq S$. This assumption is implied by the second property of SPNs. From now on, we concentrate on Self-independent Petri Nets (SPNs).

We now introduce a partial order $\sqsubseteq$ on SPNs such that $N_{1} \sqsubseteq N_{2}$ iff the following three conditions hold: 
1. $N_{1} \subseteq N_{2}$ (i.e. $\left.S_{1} \subseteq S_{2}, E_{1} \subseteq E_{2}, T_{1} \subseteq T_{2}, L_{1} \subseteq L_{2}\right)$;

2. ${ }^{\circ} N_{1} \subseteq{ }^{\circ} N_{2}$;

3. $\forall x, y \in N_{1} . x T_{2} y \Rightarrow x T_{1} y$.

The third condition implies that the projection of $N_{2}$ to $N_{1}$ coincides with $N_{1}$ (i.e. $\left.N_{2} \Upsilon_{N_{1}}=N_{1}\right)$. This means that the prefix closure implies the partial order. Hence, the least upper bound $U N_{k}$ of an $\omega$-chain $\left\{N_{k}\right\}$ is $U N_{k}=U N_{k}$, and the least element $\perp$ is $\langle\{\bullet\}, \emptyset, \emptyset, \emptyset\rangle$. Therefore, our SPNs form a cpo (complete partial order) (Plotkin, 1985).

\section{Constructable SPNs}

Let $\ell$ range over $L a b, \ell$ and $\bar{\ell}$ represent an input label and an output label respectively. Note that ${ }^{-}$is the complementary function over Lab. We give constructions for SPNs below.

1. (bottom) $\perp=<\{\bullet\}, \emptyset, \emptyset, \emptyset>$;

2. (sequence or prefix) $\ell . N={ }_{\text {def }}<\{s\} \cup S,\{e\} \cup E,\{<s, e>\} \cup\left\{<e, s^{\prime}>\mid s^{\prime} \in\right.$ $\left.\left.{ }^{\circ} N\right\} \cup T, L \cup\{\langle e, \ell\rangle\}\right\rangle$, where $N \neq \emptyset$, and $s$ and $e$ are not in $N$;

3. (non-deterministic composition) $N_{1}+N_{2}=d_{\text {def }}<S, E_{1} \cup E_{2}, T, L_{1} \cup L_{2}>$, where $N_{i} \neq \emptyset(i=1,2)$ are disjoint, $S={ }_{\text {def }}\left(S_{1}-{ }^{\circ} N_{1}\right) \cup\left(S_{2}-{ }^{\circ} N_{2}\right) \cup\left({ }^{\circ} N_{1} \times{ }^{\circ} N_{2}\right)$ and $T==_{\text {def }}\left\{\langle s, e>| s=\left\langle s_{1}, s_{2}>\wedge\left(<s_{1}, e>\in T_{1} \vee<s_{2}, e>\epsilon T_{2}\right) \wedge s_{i} \in{ }^{\circ} N_{i}(i=\right.\right.$ $1,2)\} \cup T_{1}\left\lceil\left(S_{1}-^{\circ} N_{1}\right) \times E_{1} \cup E_{1} \times\left(S_{1}-^{\circ} N_{1}\right) \cup T_{2}\left\lceil\left(S_{2}-^{\circ} N_{2}\right) \times E_{2} \cup E_{2} \times\left(S_{2}-{ }^{\circ} N_{2}\right) ;\right.\right.$

4. (parallel composition) $N_{1} \mid N_{2}={ }_{\text {def }}<S_{1} \cup S_{2}, E_{1} \cup E_{2} \cup \operatorname{prod}, T_{1} \cup T_{2} \cup$ conc, $L_{1} \cup$ $L_{2} \cup$ comm $>$, where $N_{1}$ and $N_{2}$ are disjoint, and prod $=_{\text {def }}\left\{e=\left\langle e_{1}, e_{2}\right\rangle \epsilon\right.$ $\left.E_{1} \times E_{2} \mid L_{1}\left(e_{1}\right)=\overline{L_{2}\left(e_{2}\right)} \neq \tau\right\}$, comm $=_{\text {def }}\{\langle e, \tau\rangle \mid e \in$ prod $\}$, and conc $=_{\text {def }}$ $\left\{\left\langle s_{1}, e\right\rangle,\left\langle s_{2}, e\right\rangle,\left\langle e, s_{1}^{\prime}\right\rangle,\left\langle e, s_{2}^{\prime}\right\rangle \mid e=<e_{1}, e_{2}>\in \operatorname{prod} \wedge s_{1} \in{ }^{\bullet} e_{1} \wedge s_{2} \in\right.$ $\left.\bullet e_{2} \wedge s_{1}^{\prime} \in e_{1}^{\bullet} \wedge s_{2}^{\prime} \in e_{2}^{\bullet}\right\}$

5. (synchronizer) $N \backslash \ell$ will be defined later;

6. (recursive operator) $\mu$ or fix (fixpoint operator).

To motivate the definition of the synchronizer (say $N \backslash \ell$ ), we display an example. Consider Figure 7 in the previous section. The intuitive meaning of $\left(N_{1} \mid N_{2}\right) \backslash \ell$ is to enforce that all the communications on port $\ell$ must be synchronized. The effect of such enforcement is demonstrated in Figure 11. By referring to Figure 7, we know that the events $e_{1}$ and $e_{2}$ are cut off after synchronization in Figure 11.

Also, there is no event which follows the state $s_{4}$, i.e. the events $e_{3}$ and $e_{5}$ and the state $s_{5}$ are cut off as well. In contrast, there may be events which follow the state $s_{2}$.

In order formally to capture synchronization, we have to introduce another concept, called well-rooted SPNs.

Let $N^{[0]}={ }_{\operatorname{def}}{ }^{\circ} N(\subseteq S), N^{[2 i+1]}={ }_{\operatorname{def}}\left\{\left.e \in E\right|^{\bullet} e \subseteq \bigcup_{k=0}^{i} N^{[2 k]}\right\}, N^{[2 i+2]}={ }_{\operatorname{def}}\{s \in$ $\left.\left.S\right|^{\bullet} s \cap N^{[2 i+1]} \neq \emptyset\right\}$, and $N^{*}={ }_{d e f}<S^{*}, E^{*}, T^{*}, L^{*}>$ where $S^{*}=\bigcup N^{[2 i]}, E^{*}=\bigcup N^{[2 i+1]}$, 


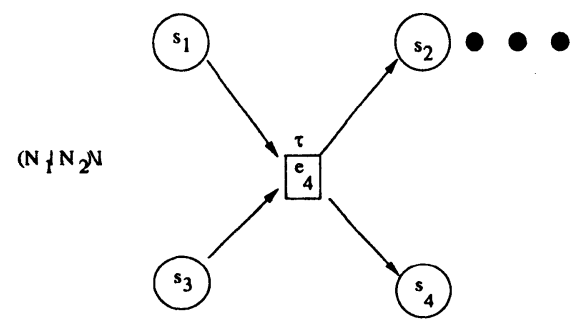

Figure 11: the net of $\left(N_{1} \mid N_{2}\right) \backslash \ell$

$T^{*}=T \Gamma_{\left(S^{*} \times E^{*}\right) \cup\left(E^{*} \times S^{*}\right)}$ and $L^{*}=L\left\lceil_{E^{*}}\right.$. We say that a SPN, $N$, is well-rooted iff $N=N^{*}$.

Informally, the idea of well-rooted nets is similar to the idea of a well-known property in set theory, viz. well-foundness. $N$ is well-rooted means that if $x \in N$, then $x$ can be reached by $\mathrm{a}(\mathrm{n})$ (arbitrary) finite number of steps (or executions or evolutions) from the roots ${ }^{\circ} N$. In other words, a well-rooted $N$ says that every state in $N$ is reachable. We consider here only the well-rooted SPNs.

Lemma 2.1: The property of well-rootedness is an invariant of the constructions of prefixes, non-deterministic compositions and parallel compositions.

For a well-rooted $N$, we define $N \backslash \ell={ }_{\text {def }}\left\langle S^{\prime}, E^{\prime}, T^{\prime}, L^{\prime}\right\rangle$, where $N^{(0)}={ }_{\text {def }}{ }^{\circ} N, N^{(2 i+1)}{ }_{d_{d e f}}$ $\left\{\left.e \in E\right|^{\bullet} e \subseteq \bigcup_{k=0}^{i} N^{(2 k)} \wedge L(e) \neq \ell, \bar{\ell}\right\}, N^{(2 i+2)}={ }_{d e f}\left\{\left.s \in S\right|^{\bullet} s \cap N^{(2 i+1)} \neq \emptyset\right\}$, $S^{\prime}=\bigcup N^{(2 i)}, E^{\prime}=\bigcup N^{(2 i+1)}, T^{\prime}=T \Gamma_{\left(S^{\prime} \times E^{\prime}\right) \cup\left(E^{\prime} \times S^{\prime}\right)}$, and $L^{\prime}=L \Gamma_{E^{\prime}}$. Thus, the result of Lemma 2.1 can be extended to include synchronizers.

Lemma 2.2: The well-rooted property is an invariant of the constructions mentioned in Lemma 2.1 with the extra constructions of synchronizers.

Also, we have that the well-rooted property is closed under least upper bounds. Formally,

Lemma 2.3: Let $\left\{N_{i}\right\}$ be an $\omega$-chain of SPNs, if $N_{i}$ is well-rooted for every $i$, then $\cup N_{i}$ is well-rooted, also.

Therefore, the result of Lemma 2.2 can be extended to include all constructions of the nets.

Theorem 2.4: The well-rooted property is an invariant of all the constructions of the nets.

The key point of the proof for Theorem 2.4 is that all the constructions are monotonic and continuous. Therefore, the fixpoints always exist when we apply $\mu$ (or $f i x$ ) to all the possible combinations of constructions, and the other constructions naturally follow from the previous lemmas.

It is not hard to prove that all constructable nets are self-independent. Furthermore, if we regard every root of a net as a process and syntactically exclude the instance of constructions like $N_{1}+\left(N_{2} \mid N_{3}\right)$, then every process is self-independent in constructable nets. That is, every token can move along constructable nets without getting stuck if we consider each root to be initially marked. This is the origin of the name of self- 
independent Petri nets comes from.

\section{Isomorphic Classes of SPNs ([SPN])}

In order that the non-deterministic compositions of $N_{1}+N_{2}$ and of $N_{2}+N_{1}$ can be regarded as identical, we must consider isomorphic classes of SPNs. First, let us give a definition for net isomorphism. $N_{1}$ is isomorphic to $N_{2}$, written as $N_{1} \cong N_{2}$, iff there is a bijection $\phi: N_{2} \rightarrow N_{1}$ such that

1. $s \in S_{2} \Leftrightarrow \phi(s) \in S_{1}$,

2. $e \in E_{2} \Leftrightarrow \phi(e) \in E_{1}$,

3. $\left\langle x, y>\epsilon T_{2} \Leftrightarrow<\phi(x), \phi(y)>\in T_{1}\right.$, and

4. $L_{2}=L_{1} \circ \phi$.

It is not difficult to see that the isomorphic relation between SPNs is an equivalence relation. Then, we define the correpsonding equivalence class over the SPNs as: $[N]=_{\text {def }}$ $\left\{N^{\prime} \in S P N \mid N^{\prime} \cong N\right\}$. We use $[\mathrm{SPN}]$ to denote the set of all the equivalence classes.

Lemma 3.1: The following three definitions are equivalent: for all $N_{i}(i=1,2)$

1. $\left[N_{1}\right] \unlhd_{1}\left[N_{2}\right]$ iff $\exists N_{2}^{\prime} \in\left[N_{2}\right] \cdot N_{1} \sqsubseteq N_{2}^{\prime}$;

2. $\left[N_{1}\right] \unlhd_{2}\left[N_{2}\right]$ iff $\exists N_{1}^{\prime} \in\left[N_{1}\right] . N_{1}^{\prime} \sqsubseteq N_{2}$;

3. $\left[N_{1}\right] \unlhd_{3}\left[N_{3}\right]$ iff $\exists N_{1}^{\prime} \in\left[N_{1}\right] . \exists N_{2}^{\prime} \in\left[N_{2}\right] . N_{1}^{\prime} \sqsubseteq N_{2}^{\prime}$.

We naturally want to extend the partial order $\subseteq$ from SPNs to $[S P N] \mathrm{s}$, denoted by $\unlhd$. Because of Lemma 3.1 , we can relate the order $\unlhd$ to any one of $\unlhd_{i}(i=1,2,3)$ as we require. However, $\unlhd$ is not, in general, a partial order but a pre-order. This is due to symmetricity, i.e. $\left[N_{1}\right] \unlhd\left[N_{2}\right] \unlhd\left[N_{1}\right]$ does not imply $\left[N_{1}\right]=\left[N_{2}\right]$.

In order to present the non-partial-order problem more clearly, let $\preceq$ be a partial order in an arbitrary $X$ and $\sim$ be an isomorphism on $X$. Then, we say that a partial order $\preceq$ in $X$ has a well-extended property (on $X$ over $\sim)$ if

$$
\forall x_{1}, x_{2} \in X . x_{1} \preceq x_{2} \wedge x_{1} \sim x_{2} \Rightarrow x_{1}=x_{2} .
$$

It is obvious that $\unlhd$ is a partial order if $\subseteq$ has the well-extended property. Unfortunately, $\sqsubseteq$ does not, in general, have the well-extended property. Therefore, it is not hard to conclude that $\unlhd$ can not be a partial order in SPNs in general. An example to show that $\sqsubseteq$ does not have the well-extended property is shown below, see Figure 12.

Let $N_{i}=<S_{i}, E_{i}, T_{i}, L_{i}>(i=1,2)$ and

1. $S_{i}=\left\{s^{i}\right\} \cup\left\{s_{n, k}^{i} \mid((i=1) \wedge(n \geq 1) \wedge(1 \leq k \leq 2 n)) \vee((i=2) \wedge(n \geq 1) \wedge(1 \leq k \leq\right.$ $2 n+1))\}$;

2. $E_{i}=\left\{e^{i}\right\} \cup\left\{e_{n, k}^{i} \mid((i=1) \wedge(n \geq 1) \wedge(1 \leq k \leq 2 n-1)) \vee((i=2) \wedge(n \geq 1) \wedge(1 \leq\right.$ $k \leq 2 n))\}$; 

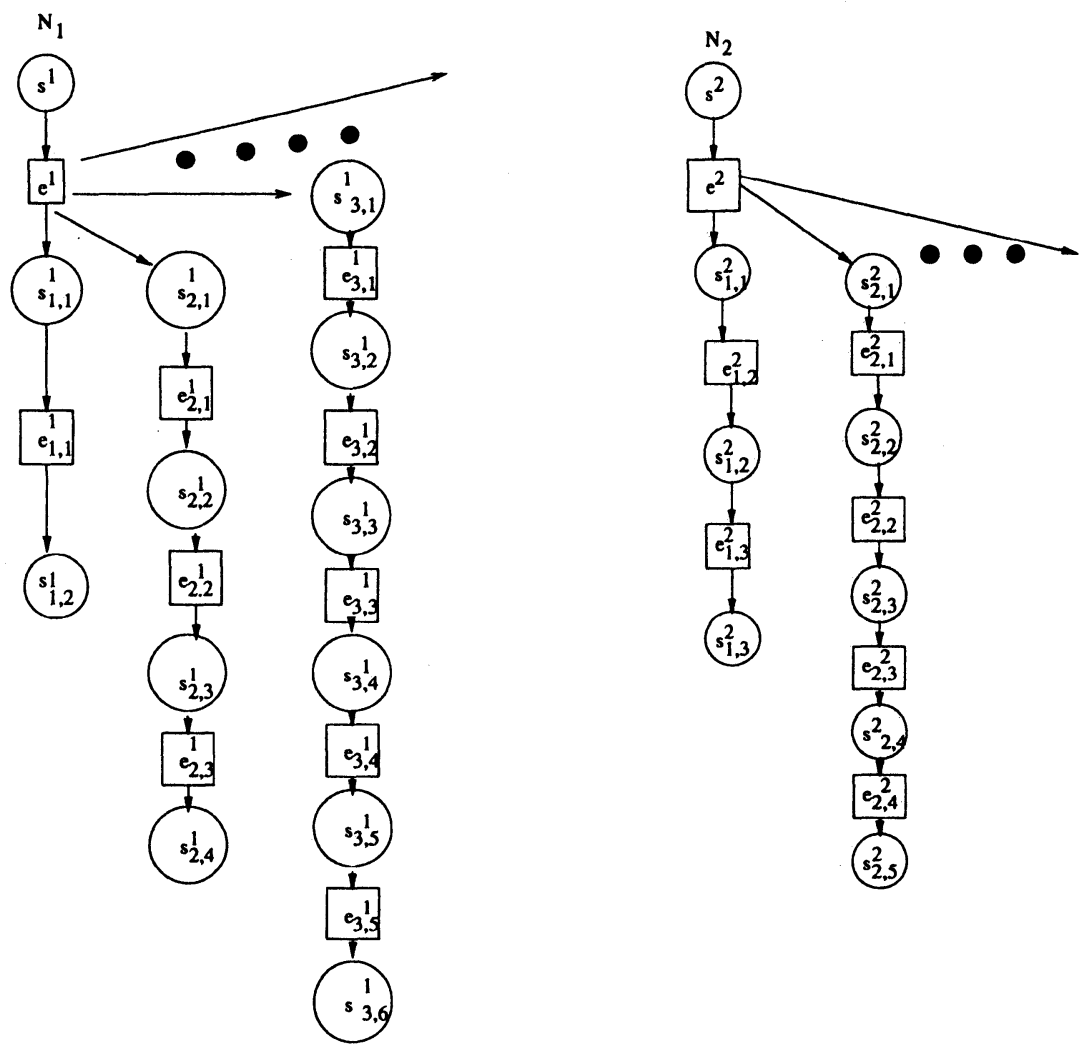

Figure 12: an example of not having the well-extended property

3. $\left.T_{i}=\left\{<s^{i}, e^{i}\right\rangle\right\} \cup\left\{<e^{i}, s_{n, 1}^{i}>\mid n \geq 1\right\} \cup\left\{<s_{n, k}^{i}, e_{n, k}^{i}>,<e_{n, k}^{i}, s_{n, k+1}^{i}>\mid((i=\right.$ 1) $\wedge(n \geq 1) \wedge(1 \leq k \leq 2 n-1)) \vee((i=2) \wedge(n \geq 1) \wedge(1 \leq k \leq 2 n))\}$;

4. $L_{i}=\left\{<e^{i}, \alpha>\right\} \cup\left\{<e_{n, k}^{i}, \beta>\mid((i=1) \wedge(n \geq 1) \wedge(1 \leq k \leq 2 n-1)) \vee((i=\right.$ 2) $\wedge(n \geq 1) \wedge(1 \leq k \leq 2 n))\}$.

It is obvious that $N_{1}$ is not isomorphic to $N_{2}$. However, we still have to check whether or not there exist $N_{1}^{\prime}$ and $N_{2}^{\prime}$ as required in $\unlhd_{3}$ in Lemma 3.1. This is done by considering an embedding of $N_{1}$ into $N_{2}$ and conversely $N_{2}$ into $N_{1}$.

Thus, in order that $\unlhd$ be a partial order, we need to restrict the SPNs to a certain subcollection. This sub-collection must be rich enough to accommodate CCS. The restriction we derive is the finitely-branched condition for SPNs, which is now introduced.

Let $N$ be a SPN. $N$ is said to be finitely-branched (or to be a fSPN) if $N$ has the following three properties:

1. for roots $\left|{ }^{\circ} \mathrm{N}\right|<\infty$;

2. $\forall e \in E .\left|e^{\bullet}\right|<\infty$; and 
3. for events $\forall i \geq 0 .\left|N^{[2 i+1]}-N^{[2 i-1]}\right|<\infty$ where $N^{[-1]}=\emptyset$.

These three conditions restrict SPNs to fSPNs such that the increase of the numbers of branches for each execution (or evolution) is limited to arbitrary finite numbers.

Since a finite set is identical to another finite one if one is a subset of the other and if there exists a 1-1 mapping between the elements of the two sets, fSPNs naturally hold the well-extended property. We formally express this result as a lemma.

Lemma 3.2: $\sqsubseteq$ is well-extended in ISPNs.

Therefore,

Theorem 3.3: $\unlhd$ is a partial order in [FSPN].

We now know that the finitely-branched conditions are sufficient to guarantee that $\unlhd$ be a partial order. However, the necessity of these conditions needs to be demonstrated. On the other hand, we can show that they are not too restrictive in the sense that there are examples where the symmetricity does not hold. Such an example has been given earlier in demonstrating that the well-extended property does not hold in general. We will give another example in the proof of Lemma 3.4. In this sense, we claim that the finitely-branched conditions are necessary for $\unlhd$ to be a partial order.

Lemma 3.4: There are $\left[N_{1}\right]$ and $\left[N_{2}\right]$ such that $\exists N_{1}^{\prime} \in\left[N_{1}\right]$ and $\exists N_{2}^{\prime} \in\left[N_{2}\right]$ and they satisfy the following three conditions:

1. $N_{1} \subseteq N_{2}^{\prime}$, i.e. $\left[N_{1}\right] \unlhd_{1}\left[N_{2}\right]$;

2. $N_{2} \subseteq N_{1}^{\prime}$, i.e. $\left[N_{2}\right] \unlhd_{1}\left[N_{1}\right]$; and

3. $N_{1}$ and $N_{2}$ are not isomorphic to each other, i.e. $\left[N_{1}\right] \neq\left[N_{2}\right]$.

\section{Proof}

The example about the well-extended property can be used here. However, this only shows that $\forall e \in E .\left|e^{\bullet}\right|<\infty$ is necessary. Removing the prefix from the example, it will be shown that $\left.\right|^{\circ} N \mid<\infty$ is necessary. For the other case, we provide another example. Assume that $\exists j .\left|N^{[2 j+1]}-N^{[2 j-1]}\right| \nless \infty$ and $\left|N^{[2 j+2]}-N^{[2 j]}\right| \nless \infty$ (the conjunction is because of the nets' characteristics of no redundancy), say $j=0$. Readers are encouraged to draw a graphical representation of the following example, since a diagram can aid understanding.

Let $N_{i}=<S_{i}, E_{i}, T_{i}, L_{i}>(i=1,2)$, where

1. $S_{i}=\left\{s^{i}\right\} \cup\left\{s_{k}^{i} \mid k \in N a t-\{0\}\right\}$;

2. $E_{i}=\left\{e_{k, n}^{i} \mid((i=1) \wedge(1 \leq n \leq 2 k-1)) \vee((i=2) \wedge(1 \leq n \leq 2 k))\right\}$;

3. $T_{i}=\left\{<s^{i}, e_{k, n}^{i}>,<e_{k, n}^{i}, s_{k}^{i}>\mid((i=1) \wedge(1 \leq n \leq 2 k-1)) \vee((i=2) \wedge(1 \leq n \leq\right.$ $2 k))\}$; and

4. $L_{i}=\left\{<e_{n, k}^{i}, \alpha^{i}>\mid((i=1) \wedge(1 \leq n \leq 2 k-1)) \vee((i=2) \wedge(1 \leq n \leq 2 k))\right\}$.

It is obvious that $N_{1}$ is not isomorphic to $N_{2}$ (see Figure 13). 


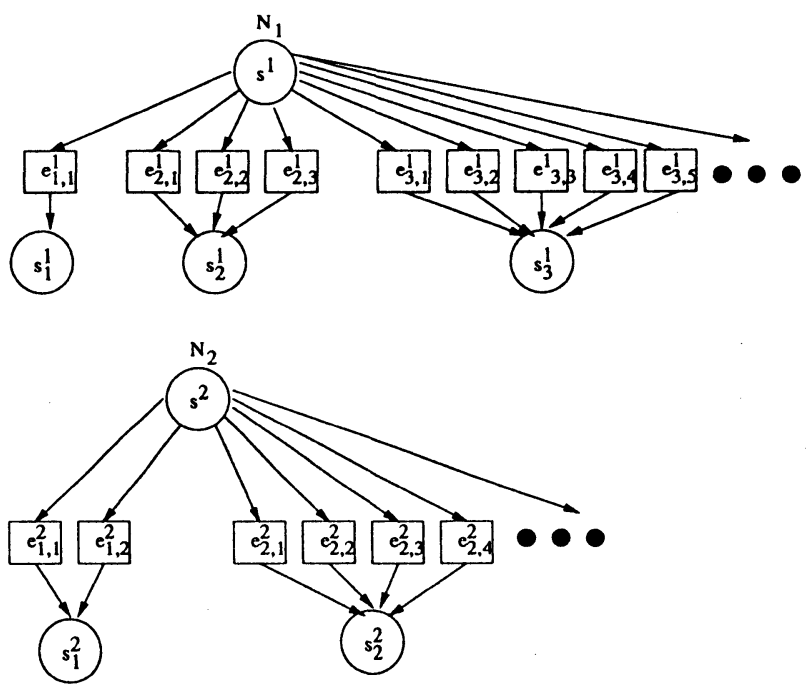

Figure 13: non-isomorphic nets

However, we still need to check whether there exist $N_{1}^{\prime}$ and $N_{2}^{\prime}$ as required. This is achieved by considering an embedding $N_{1}$ into $N_{2}$ and conversely $N_{2}$ into $N_{1}$.

For an $\omega$-chain $\left\{\left[N_{k}\right]\right\}$ in [fSPN], its least upper bound is:

$$
\sqcup\left[N_{k}\right]=\left[\bigcup N_{k}^{\prime}\right]
$$

where $\forall k . N_{k}^{\prime} \in\left[N_{k}\right] \wedge N_{k}^{\prime} \sqsubseteq N_{k+1}^{\prime}$. So, [fSPN] forms a cpo.

Naturally, we can extend the constructions of the last section (Section 3) to [fSPN] as follows:

1. $\alpha \cdot[N]=[\alpha \cdot N]$

2. $\left[N_{1}\right]+\left[N_{2}\right]=\left[N_{1}+N_{2}\right]$

3. $\left[N_{1}\right] \mid\left[N_{2}\right]=\left[N_{1} \mid N_{2}\right]$

4. $[N] \backslash \alpha=[N \backslash \alpha]$.

Since these constructions on [fSPN] are defined by their counterparts in fSPNs, their monotonicities and continuities follows easily. However, there is no trivial way to extend recursive definitions to isomorphic classes. For example, $x \Rightarrow x \mid \alpha . \perp$ where $x$ is an identifier for nets. That is, $\mu$ is not closed in fSPNs. So we have further to restrict constructions to certain instances.

By the application of the well-constructed restriction, a unary construction $F$ (or functor) of fSPNs is said to be well-constructed if

$$
\exists K . \forall k \geq K .\left.\right|^{\circ} F^{k}([<\{\bullet\}, \emptyset, \emptyset, \emptyset>])|=|^{\circ} F^{k+1}([<\{\bullet\}, \emptyset, \emptyset, \emptyset>]) \mid<\infty .
$$


The well-constructed condition for nets excludes the possibility of increasing the number of roots of a constructable net to an infinite number, i.e. a well-constructed $F$ cannot increase the number of roots of a net to an arbitrary large value by recursively applying $F$. So we have that

Theorem 3.5: For any well-constructed $F, \mu x .(\lambda x . F)$ is closed in [fSPN].

The proof is omitted. However, the key point of the proof is to show that a series of numbers of root states (to the least element) converges by recursively applying $F$, and so the least fixed point of $F$ is an fSPN. A similar theorem (to Theorem 3.5) for mutual recursive functors $\vec{F}$ can also be established, but we do not give its details.

Since it can be easily checked that other constructions are closed in [fSPN], the net constructions in section 3 under the well-constructed restriction are also closed in [fSPN]. Therefore, it is safe to interprete CCS terms in the well-constructed [fSPN], i.e. the wellconstructed [fSPN] is an adequate denotational semantics model for CCS provided that the CCS terms are well-guarded.

\section{Discussion and Conclusions}

From the categorical point of view, the constructions + and $\mid$ are consistent with their sum and product in the Petri nets category (Winskel, 1987) except that $\mid$ is a restricted product which only affects the events with complementary labels. This shows that the intuition of the asynchrony from implementation has a categorical background. Also, it is worth mentioning that the asynchrony is by its nature deadlock-free.

We should point out that the synchrony and the asynchrony in this paper are different from those of Milner (1984). His synchrony implies the existence of a universal clock and his asynchrony implies the non-existence of such a clock.

In this paper, we propose that the asynchronous mechanism for communication should be considered as a primitive for semantic models of distributed systems in contrast to the synchronous mechanism (hand-shaking) of CSP (Hoare, 1985) or of CCS (Milner, 1989). By doing so, we present an approach to producing a deadlock-free system, and give a denotational model for CCS in a non-interleaving fashion by means of self-independent Petri Nets.

Another advantage from the asynchrony not having been exploited in this paper is to incorporate broadcasting communication into the model. This will be the subject of future investigation.

Comparing constructable nets (or cSPNs for short) with fSPNs, we understand that the collection of cSPNs is more restrictive than the collection of fSPNs. In the light of this observation, the condition for net constructions to be both finitely-branched and well-constructed may be over restrictive. This issue requires more attention.

\section{Acknowledgement}

The first author would like to thank G. Plotkin for his insight of SPNs. We would like to thank Prof. M. Clint of the Queen's University of Belfast and four anonymous referees for their constructive comments on early version of this paper and Y.Sato of Hiroshima City 
University for his support in using computer facilities. This work is supported in part by the Ministry of Education of Japan under Joint Research Grant-in-Aid for International Scientific Research FM-ISEE (08044167), by Hiroshima City University under Hiroshima City University Grant for Special Academic Research (International Studies) SCS-FM (A440), and by NIDevR of the Queen's University of Belfast. We would like to express our special gratitude to these organizations for their support.

\section{References}

[1] G. Boudol and I. Castellani (1987), "On the Semantics of Concurrency: partial order and transition systems", TAPSOFT'87, Lecture Notes in Computer Science, Vol. 249, Springer-Verlag, 1987.

[2] U. Goltz and A. Mycroft (1984), "On the Relationship of CCS and Petri Nets", Lecture Notes in Computer Science, Vol. 172, Springer-Verlag, 1984.

[3] C. A. R. Hoare (1985), "Communicating Sequential Processes", Prentice-Hall International, 1985.

[4] L. Lamport, R. Shostak, and M. Pease (1982), "The Byzantine General Problem", ACM Trans. on Prog. Lang. and Syst., Vol.4, No.3, July 1982.

[5] Robin Milner (1984), "Calculi of Synchrony and Asynchrony", Journal of Theoretical Computer Science, 1984.

[6] Robin Milner (1989), "Communication and Concurrency", Prentice-Hall International, 1989.

[7] M. Neilsen, G. Plotkin and G. Winskel (1980), "Petri Nets, Event Structures and Domains, Part 1", Journal of Theoretical Computer Science, 1980; also appears in internal report of Department of Computer Science, University of Edinburgh, CSR-47-79, November 1979.

[8] J. L. Peterson (1981), "Petri Nets Theory and the Modelling of Systems", PrenticeHall, Englewood Cliffs, N. J., 1981.

[9] C. A. Petri (1976), "General Net Theory", Communication Disciplines, ed. B. Show, Proc. Joint IBM and University of Newcastle Seminar, 1976.

[10] G. D. Plotkin (1985), "Domain Theory", Lecture notes for the postgraduates of the Department of Computer Science, University of Edinburgh, 1985/6.

[11] Yong Sun and Hongji Yang (1996), "Communication mechanism independent protocol specification based on CSP: a case study", in the proceedings of the 22nd Euromicro conference: Beyond 2000: Hardware and Software Design Strategies, IEEE Computer Society, 2-5 September, Pregue, Czech Republic, 1996.

[12] G. Winskel (1983), "Event Structure Semantics for CCS and Related Languages", Lecture Notes in Computer Science, Vol.140, Springer-Verlag, 1983.

[13] G. Winskel (1987), "Petri Nets, Algebras, Morphisms, and Compositionality", Journal of Information and Computation, Vol.72, 1987. 


\section{Biography}

Yong Sun was born at Nanning, Guangxi Province, P R China on 1st September 1961. He received his BSc, MSc and $\mathrm{PhD}$ (all in Computer Science) from Peking University in 1982, the Institute of Computing Technology (Chinese Academy of Sciences, Beijing) in 1985, and the University of Edinburgh in 1992, respectively. And he has over 20 publications in refereed journals and conferences. He is a member of IEEE Computer Society, the Association of Computing Machinery, the European Association of Theoretical Computer Science. At the present, he is a Lecturer in Computer Science of the Queen's University of Belfast, Northern Ireland.

Shaoying Liu was born in Shaanxi Province, P R China on 25th April 1960. He received his BSc and MSc in Computer Science in 1982 and 1987 respectively from Xi'an JiaoTong University, P R China, his PhD in Formal Methods of Software Engineering in 1992 from the University of Manchester, United Kingdom. He has served as general chair, coprogram chair, and program committee members for many international conferences and has over 30 publications in refereed journals and conferences. He is a member of IEEE Computer Society, IEICE Japan and the Order of International Fellowship (MOIF). At present, he is an Associate Professor in Computer Science of Hiroshima City University in Japan.

Mitsuru Ohba is a Prefessor of Computer Science Department, Hiroshima City University since April 1994. Before that, he was a senior researcher of IBM at Systems Engineering Laboratory, Tokyo, Japan. He received both BSc and MS from Aoyama Gakuin University, Tokyo, Japan, in 1971 and 1973 respectively. His present interests are in collaboration in a distributed environment, software testing and application of software metrics for software process improvements. 